\title{
Colchicine Treatment for Tracheobronchial Amyloidosis
}

\author{
Arturo Morales ${ }^{a}$ Marizell Parib ${ }^{b}$ Rosa López-Lisbona ${ }^{b}$ Noelia Cubero ${ }^{b}$ \\ Jordi Dorcab Antoni Rosell ${ }^{b}$ \\ aDepartamento de Enfermedades Respiratorias, Facultad de Medicina, Pontificia Universidad Católica de Chile,

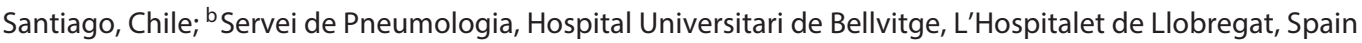

\section{Established Facts}

- Tracheobronchial amyloidosis is a rare disease that can lead to considerable morbidity and mortality.

- There is no randomized trial of treatment directed specifically to this disease.

\section{Novel Insights}

- Colchicine may have an important role in the long-term control of this form of localized amyloidosis, which differs from the response observed when the same treatment is given for systemic amyloidosis.

\section{Key Words}

Tracheobronchial amyloidosis · Treatment · Colchicine · Case report · Laryngeal amyloidosis · Bronchoscopy

\begin{abstract}
Tracheobronchial amyloidosis is an infrequent disease characterized by the deposition of proteinaceous material in the tracheobronchial tree. The disease generally has a high morbidity and variable mortality in the years following diagnosis. There is no consensus on the optimal treatment. We report a case of a 63-year-old woman who presented with a diffuse tracheobronchial amyloidosis associated with laryngeal involvement, which required a percutaneous tracheostomy due to high-grade subglottic stenosis, with no evidence of systemic amyloidosis. After treatment exclusively with col-
\end{abstract}

chicine, she had a complete resolution of the stenotic area, with a very good response from the tracheobronchial amyloidosis disease, with only minor yellow plaques persisting. The patient has remained asymptomatic in the next 4 years of follow-up, with no evidence of endoscopic progression. This is the first documented case of this kind of response of tracheobronchial amyloidosis to colchicine treatment alone. A review of the available literature is presented.

(c) 2016 S. Karger AG, Basel

\section{Introduction}

Amyloidosis is a rare disease that results from an abnormal extracellular tissue deposition of proteinaceous material, conforming insoluble fibrils of low molecular

\section{KARGER}

E-Mail karger@karger.com www.karger.com/res (c) 2016 S. Karger AG, Base

0025-7931/16/0913-0251\$39.50/0

Antoni Rosell, MD, PhD, Respiratory Endoscopy Unit, Pulmonology Department 
Fig. 1. Chest $\mathrm{CT}$ at the initial visit. Black arrows show circumferential wall thickening at the trachea (a) and main bronchus (b).
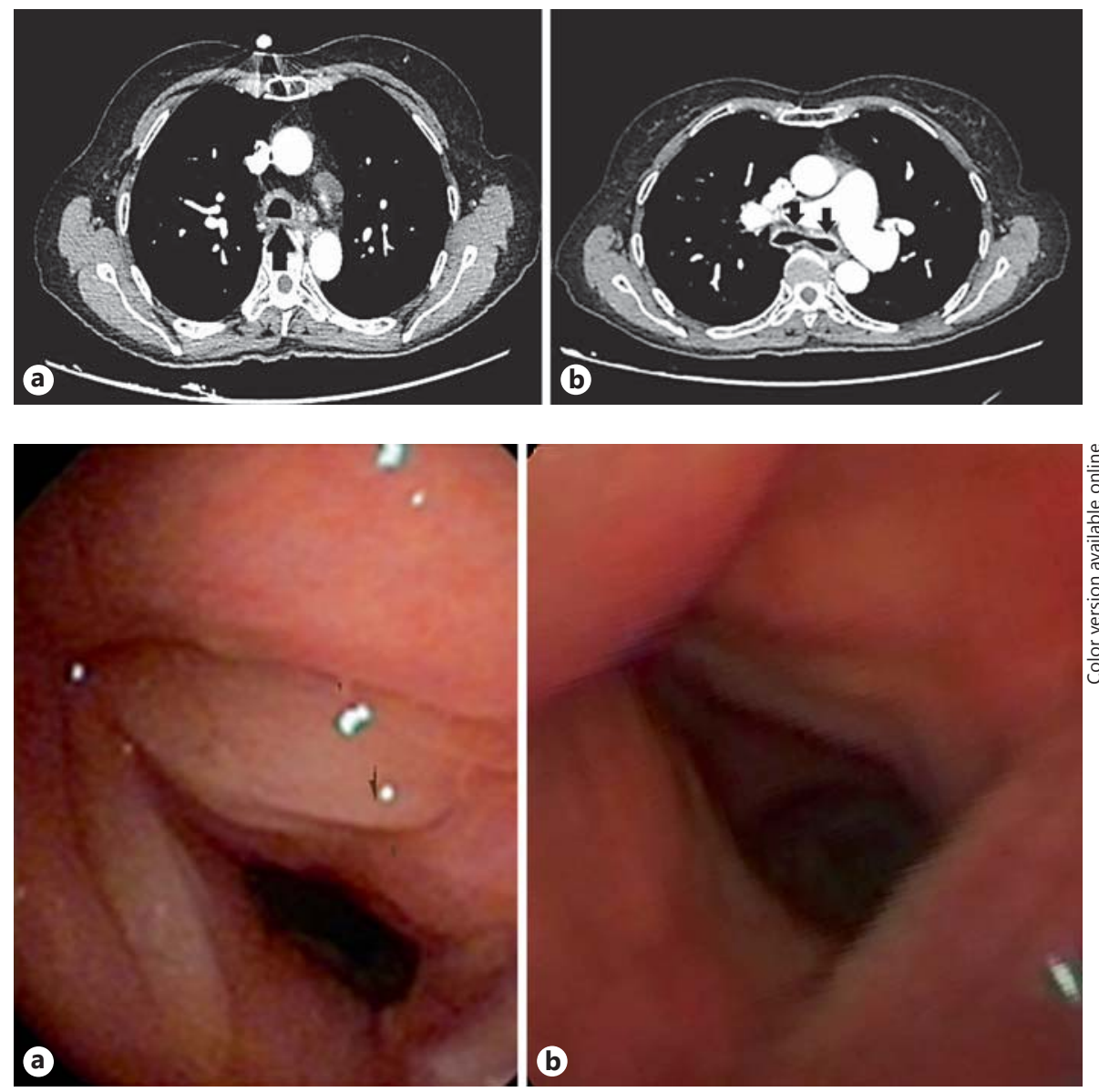

Fig. 2. Bronchoscopic images of the subglottic stenosis (laryngeal view) at the initial visit (a) and 8 months later (b). weight, which leads to organ damage [1]. The disease can be systemic, characterized by widespread organ deposition, or localized. Tracheobronchial amyloidosis (TBA) is one of the multiple manifestations of localized pulmonary amyloidosis. Respiratory symptoms are nonspecific, diagnosis is supported by bronchoscopy findings and confirmed by birefringence of Congo red-stained tissue under polarized light microscopy [2]. TBA is an uncommon disease [3] and its treatment is a matter of constant debate in the scant series published. We report a case of a middle-aged woman with TBA with an excellent response to colchicine treatment.

\section{Case Report}

A 63-year-old woman was diagnosed of TBA 10 years ago, based on a history of repeated episodes of pneumonia and positive staining for Congo red and substance $\mathrm{P}$ in bronchial tissue. She had been asymptomatic since then, but in the last 2 months before the initial visit she reported dry cough and hoarseness, and in the last week minor hemoptysis, fever up to $38.2^{\circ} \mathrm{C}$, and asthenia, with no dyspnea. She was eupneic, with a pulse oxymetry of $97 \%$ at room air. Her physical examination was unremarkable. Blood count, kidney and liver function, as well as urinary sediment were all normal. A chest X-ray demonstrated a right perihilar radiopaque area, which was complemented with a chest computed tomography (CT) that showed a chronic residual imaging of minor cystic bronchiectasis with mucous impactation. In addition, regular concentric tracheal and bronchial wall thickening was identified in the CT image (fig. 1). Flexible bronchoscopy performed at that time showed bilateral subcordal thickened tissue that determined a subglottic stenosis leaving $30 \%$ of free lumen (fig. 2a). Subglottic stenotic patency was assessed by subjective visual assessment by an experienced bronchoscopist. The respiratory mucosa was micronodular, friable, with an inflammatory appearance, extending from the trachea to the carina, without significant lumen reduction, all compatible with TBA (fig. 3a, b). Microbiology was negative for mycobacterium and fungi cultures. Systemic amyloidosis was ruled out with negative serum and urinary immunofixation, normal electrophoresis, a normal echocardiogram and a fat pad aspiration that showed no amyloid deposits. She had normal quantitative immunoglobulin studies. An electromyography showed no electrophysiological neuropathy. Study of DNA for familial amyloidosis was also negative. The patient was treated with a 10-day course of combined amoxicillin-clavulanate and corticosteroids at a dosage of $1 \mathrm{mg} / \mathrm{kg}$, and was started on colchicine chronic therapy 

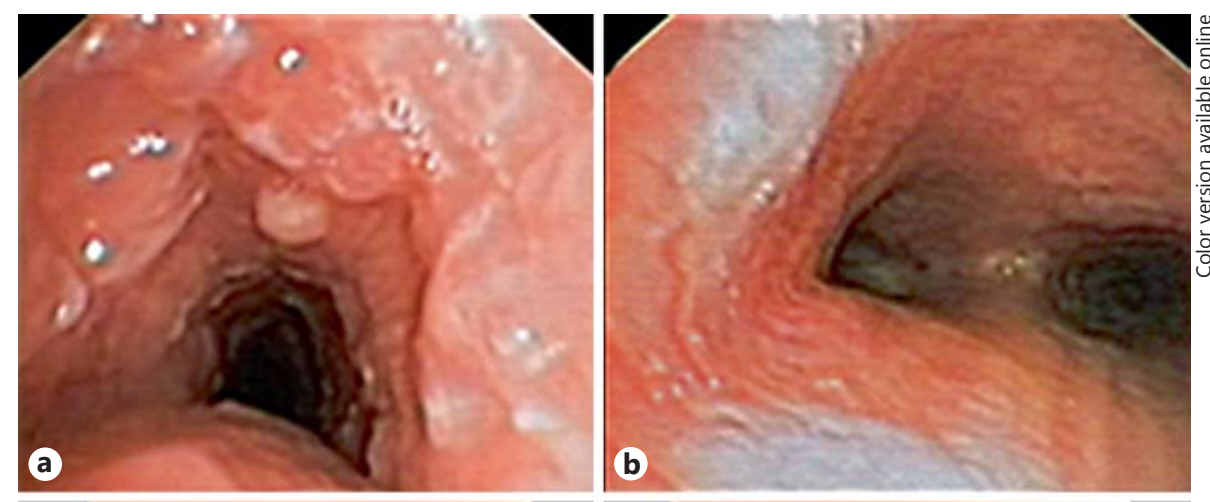

Fig. 3. Tracheobronchial disease. Bronchoscopic imaging of the trachea and main carina showing micronodular, friable, inflammatory appearance of respiratory mucosa, extending from the trachea to the carina, without significant lumen reduction at the initial visit $(\mathbf{a}, \mathbf{b})$, with a practically normal appearance after colchicine treatment $(\mathbf{c}, \mathbf{d})$.
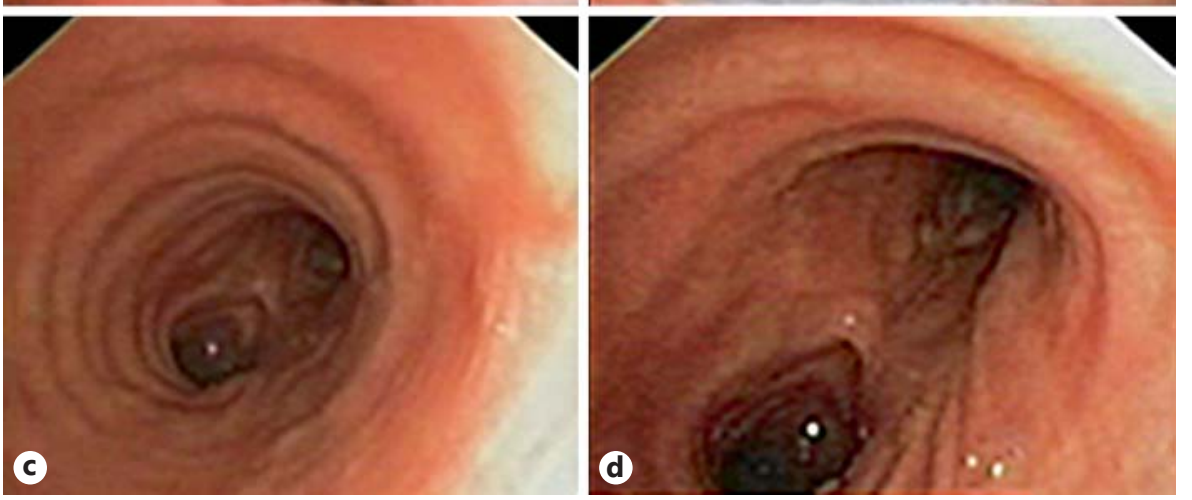

at $1 \mathrm{mg}$ /day with clinical improvement. A month later, the patient attended the emergency department with dyspnea, fever and stridor, and H1N1 flu was diagnosed. Antiviral treatment was initiated with oseltamivir $75 \mathrm{mg} /$ day. One week later, a new bronchoscopy showed progression of the subglottic submucosal thickening, leaving a clear passage of $15-20 \%$ of tracheal lumen, with lesions in the right principal bronchus with a $20 \%$ stenosis. A formal tracheostomy was performed, and colchicine dosis was raised to 2 $\mathrm{mg} /$ day. After 4 months of colchicine treatment, a clear improvement in images and symptoms was observed. No other treatment was necessary. At 8 months, the patient was completely asymptomatic and the subglottic stenosis was completely resolved (fig. 2b), and the tracheotomy tube could be withdrawn. At flexible bronchoscopy, only small yellow plaques in the tracheal and bronchial mucosa persisted (fig. $3 \mathrm{c}, \mathrm{d}$ ). At that time, colchicine was withdrawn because of alopecia and leucopenia. The patient has been asymptomatic in the last 4 years of follow-up, with only isolated episodes of hemoptysis with viral respiratory infections. Control bronchoscopies have not shown any progression.

\section{Discussion}

TBA is one of the multiple localized manifestations of respiratory amyloidosis disease. Although a number of cases have been reported in 25- to 80-year-old patients, the disease typically is diagnosed around the fifth decade of life. Preponderance in men is described in the largest series [4-7]. The symptoms generally reported are cough, dyspnea, hoarseness and occasional hemoptysis, similar to our case $[1,4,5]$. According to published series, the definitive diagnosis is generally delayed by $8-37$ months from the onset of symptoms, with patients frequently being misdiagnosed as having asthma or chronic obstructive pulmonary disease $[4,5]$. In its pathogenesis, amyloid fibrils are generally composed by AL type, not identifying a systemic monoclonal light chain disease [8]. Some reports suggest a local production of amyloidogenic light chains by subtle local clones of lymphoplasmocytes $[1,9]$. As in our case, diagnosis is suspected by clinical, radiological and bronchoscopic findings, and confirmed by biopsy tissue that shows birefringence of Congo red-stained tissue under polarized light microscopy [2]. Chest radiography may be normal in up to $70 \%$ of patients [5], and only indirect signs of obstruction with atelectasis or volume loss may be present $[10,11]$. The chest CT of TBA patients generally exhibits tracheobronchial circumferential thickening, sometimes with calcifications, luminal narrowing, atelectasis and/or localized bronchiectasis [5, 10]. Variable patterns in pulmonary function tests have been reported depending on the site of TBA involvement [10]. Bronchoscopic appearance may be present as either diffuse submucosal plaques or local tumor-like masses, the first one being the most commonly reported [1]. Bronchoscopy probe-based confocal endomicroscopy 
obtains in vivo cellular scale images, and when it is employed over areas of TBA, 'cotton wool'-like appearance images are obtained [12].

The clinical course is highly variable. Some series reports mortality in up to $75 \%$ within 6 years from diagnosis, while in others no mortality is reported in the 7.6-year average follow-up [7]. The estimated 5-year survival for the diffuse TBA disease is $30-50 \%[5,13]$. Our patient remained asymptomatic for 10 years from initial diagnosis; this evolution has been reported in some cases $[5,14]$. The tracheobronchial progression witnessed in her case was associated with laryngeal amyloidosis. This is not surprising, since there have been previous reports of concomitant laryngeal and TBA [15], without pointing to an underlying systemic amyloidosis disease.

There is no established treatment for TBA. Due to the scant number of cases, no randomized trial has been performed. The management of primary TBA is largely dependent on symptoms. Therefore, management decisions have to be taken on an individual basis. In asymptomatic patients, no treatment is necessary and they just need to be followed up, as was the case with this patient in the 10 years since the initial diagnosis. In symptomatic patients, the options are bronchoscopic recanalization, pharmacological treatment, and/or external beam radiation. The friable nature of the amyloid lesions generally results in bleeding during procedures. In the series reported by Díaz-Jiménez et al. [7], the resection had to be stopped because of excessive bleeding in 2 out of 11 patients. Pharmacologic treatment of localized amyloidosis is only based on case report studies, with no formal randomized controlled trials available. Colchicine has been tried in some cases of TBA with different results $[5,10,14]$. A French study reported one patient treated with colchicine, reporting local dissemination after 7 years of follow-up [14]. In a Chinese series, 6 out of 64 patients were treated with colchicine, but there is no report of follow-up [5]. A randomized trial for systemic amyloidosis comparing colchicine to melphalan or corticoids or a combination of all three therapies, showed reduced mortality in the melphalanand corticoid-containing regimens [16]. However, due to the difference in pathogenesis of systemic and local amyloidosis, these results cannot be extrapolated to TBA treatment [16]. Colchicine is an inhibitor of familial Mediterranean fever amyloidosis and of mouse experimental amyloidosis $[17,18]$. It inhibits microtubular system during the metaphase and decreases monocyte and neutrophil chemotaxis [18]. Colchicine can inhibit the deposition of amyloid fibrils in organs, as has been demonstrated in mice models [17], possibly related to its anti-inflammato- ry effect. Other case reports of TBA include treatment with glucosteroids alone or in combination with colchicine and showed overall benefits in one third of cases [5]. Our patient had a great response to colchicine treatment alone, with complete regression of subglottic stenosis and significant reduction of tracheobronchial disease, but this fact cannot be attributed exclusively to pharmacologic treatment. The amyloid deposits are not static, with turnover being reported, and they can sometimes regress when the supply of fibril precursor protein is reduced [19]. It cannot be discarded that a spontaneous resolution of the disease could have happened, as has been previously reported in one isolated case [20]. We consider this last hypothesis to be extremely improbable given the extension of the disease and the scant reports in the literature.

Other treatments options include bronchoscopic recanalization of the airway. Laser treatment, mechanical debulking, balloon dilatation and/or stent placement have been reported $[5,21,22]$. In a Chinese series of 64 patients, a treatment option was required in $75 \%$ of the patients, with Nd:YAG laser and the mechanical debulking being the most common approaches. Although shortterm relief was reported, half of them had a poor response [5]. In symptomatic laryngeal amyloidosis, the treatment option is usually an endoscopic one [1], with the carbon dioxide $\left(\mathrm{CO}_{2}\right)$ and $\mathrm{Nd}$ :YAG laser approach being the most frequently reported $[21,23]$. External beam radiation therapy has been used with good results, but the exact mechanism by which it obtains its beneficial effects is unknown and evidence is only available from case-controlled studies $[12,23,24]$. Its main complication is esophagitis. Recurrence is reported in the range of $8-80$ months after treatment [23]. All the previous therapies are not exclusive and multimodal therapy was attempted in some cases $[23,24]$. In our patient, due to her excellent response to colchicine treatment, no further therapies were needed. The proximal tracheal and subglottic disease that she presented, as well as diffuse TBA disease, are known to have a poorer prognosis $[10,13]$; nevertheless, she has continued to be asymptomatic in the last 4 years.

In conclusion, we present a patient with a long-term documented regression ad integrum of her TBA, in response to colchicine treatment. To our knowledge, this is the first report of such an excellent response.

\section{Financial Disclosure and Conflicts of Interest}

The authors declare no conflicts of interest in preparing this article. 


\section{References}

1 Gillmore JD, Hawkins PN: Amyloidosis and the respiratory tract. Thorax 1999;54:444451.

$>2$ Glenner GG, Eanes ED, Page DL: The relation of the properties of Congo red-stained amyloid fibrils to the $\beta$-conformation. J Histochem Cytochem 1972;20:821-826.

$>3$ Utz JP, Swensen SJ, Gertz MA: Pulmonary amyloidosis. The Mayo Clinic experience from 1980-1993. Ann Intern Med 1996;124: 407-413.

$\checkmark 4$ Capizzi SA, Betancourt E, Prakash UB: Tracheobronchial amyloidosis. Mayo Clin Proc 2000;75:1148-1152.

$>5$ Ding L, Li W, Wang K, Chen Y, Xu H, Wang $\mathrm{H}$, et al: Primary tracheobronchial amyloidosis in China: analysis of 64 cases and a review of literature. J Huazhong Univ Sci Technolog Med Sci 2010;30:599-603.

$\checkmark 6$ Hui AN, Koss MN, Hochholzer L, Wehunt WD: Amyloidosis presenting in the lower respiratory tract. Clinicopathologic, radiologic, immunohistochemical, and histochemical studies on 48 cases. Arch Pathol Lab Med 1986;110:212-218.

7 Díaz-Jiménez J, Rodríguez A, Martínez-Ballarín J, Castro MJ, Argemi T, Manresa F: Diffuse tracheobronchial amyloidosis. J Bronchology 1999;6:13-17.

-8 Berk J, O’Regan A, Skinner M: Pulmonary and tracheobronchial amyloidosis. Semin Respir Crit Care Med 2002;23:155-165.
$>9$ Borie R, Danel C, Molinier-Frenkel V, Prevot G, Deslee G, Debray MP, et al: Tracheobronchial amyloidosis: evidence for local B-cell clonal expansion. Eur Respir J 2012;39:10421045.

10 O'Regan A, Fenlon H, Beamis J, Steele M, Skinner M, Berk J: Tracheobronchial amyloidosis. The Boston University experience from 1984 to 1999. Medicine 2000;79:69-79.

11 Prowse CB: Amyloidosis of the lower respiratory tract. Thorax 1958;13:308-320.

12 Newton R, Kemp S, Yang G, Darzi A, Sheppard M, Shah P: Tracheobronchial amyloidosis and confocal endomicroscopy. Respiration 2011;82:209-211.

13 Sommer P, Kumar G, Lipchik RJ, Patel JJ: Tracheobronchial amyloidosis managed with multimodality therapies. Ther Adv Respir Dis 2014;8:48-52.

14 Paccalin M, Hachulla E, Cazalet C, Tricot L, Carreiro M, Rubi M, et al: Localized amyloidosis: a survey of 35 French cases. Amyloid 2005; 12:239-245.

15 Thompson LD, Derringer GA, Wenig BM: Amyloidosis of the larynx: a clinicopathologic study of 11 cases. Mod Pathol 2000;13:528535.

16 Kyle RA, Gertz MA, Greipp PR, Witzig TE, Lust JA, Lacy MQ, et al: A trial of three regimens for primary amyloidosis: colchicine alone, melphalan and prednisone, and melphalan, prednisone, and colchicine. N Engl J Med 1997;336:1202-1207.
17 Shtrasburg S, Pras M, Gal R, Salai M, Livneh A: Inhibition of the second phase of amyloidogenesis in a mouse model by a singledose colchicine regimen. J Lab Clin Med 2001; 138:107-111.

18 Kucuk A, Gezer IA, Ucar R, Karahan AY: Familial Mediterranean Fever. Acta Medica (Hradec Kralove) 2014;57:97-104.

19 Hawkins PN: Studies with radiolabelled serum amyloid $\mathrm{P}$ component provide evidence for turnover and regression of amyloid deposits in vivo. Clin Sci 1994;87:289-295.

20 Hof DG, Rasp FL: Spontaneous regression of diffuse tracheobronchial amyloidosis. Chest 1979;76:237-239.

21 Alloubi I, Thumerel M, Begueret H, Baste J, Velly J, Jougon J: Outcomes after bronchoscopic procedures for primary tracheobronchial amyloidosis: retrospective study of 6 cases. Pulm Med 2012;2012:1-4.

22 Yang S, Chia SY, Chuah KL, Eng P: Tracheobronchial amyloidosis treated with rigid bronchoscopy and stenting. Surg Endosc 2003;17:658-659.

23 Truong M, Kachnic L, Grillone G, Bohrs H, Lee R, Sakai O, et al: Long-term results of conformal radiotherapy for progressive airway amyloidosis. Int J Radiat Oncol Biol Phys 2012;83:734-739.

24 Neben-Wittich MA, Foote RL, Kalra S: External beam radiation therapy for tracheobronchial amyloidosis. Chest 2007;132:262-267. 